\title{
Minimizing Redundant Messages and Improving Search Efficiency under Highly Dynamic Mobile P2P Network
}

\author{
Ajay Arunachalam*and Ohm Sornil \\ Department of Computer Science, Graduate School of Applied Statistics, National Institute of Development \\ Administration, Thailand
}

Received 7 October 2015; Accepted 25 February 2016

\begin{abstract}
Resource Searching is one of the key functional tasks in large complex networks. With the P2P architecture, millions of peers connect together instantly building a communication pattern. Searching in mobile networks faces additional limitations and challenges. Flooding technique can cope up with the churn and searches aggressively by visiting almost all the nodes. But it exponentially increases the network traffic and thus does not scale well. Further the duplicated query messages consume extra battery power and network bandwidth. The blind flooding also suffers from long delay problem in P2P networks. In this paper, we propose optimal density based flooding resource discovery schemes. Our first model takes into account local graph topology information to supplement the resource discovery process while in our extended version we also consider the neighboring node topology information along with the local node information to further effectively use the mobile and network resources. Our proposed method reduces collision at the same time minimizes effect of redundant messages and failures. Overall the methods reduce network overhead, battery power consumption, query delay, routing load, MAC load and bandwidth usage while also achieving good success rate in comparison to the other techniques. We also perform a comprehensive analysis of the resource discovery schemes to verify the impact of varying node speed and different network conditions.
\end{abstract}

Keywords: MANET, Mobile Peer-to-Peer, Flooding, Node Density

\section{Introduction}

MANETs are dynamic network which are characterized by wireless links, mobile nodes and multihop routing. In such network the nodes that are not directly connected at the Link layer can communicate through the routing layer as shown in Fig. 1.
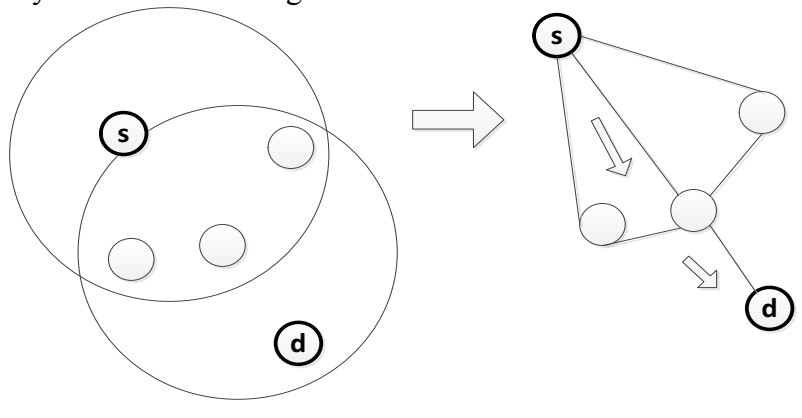

Fig.1. Example MANET topology

With frequent changes in topology due to mobility, resource discovery in such network becomes more challenging. To cope with churn, traditional searching techniques are considered. Flooding and random walk are the two widely used techniques. In flooding, the query is flooded to every node in the network while in

\footnotetext{
*E-mail address: ajay.arunachalam08@gmail.com ISSN: 1791-2377 @ 2016 Eastern Macedonia and Thrace Institute of Technology. All rights reserved.
}

random walk nodes are chosen randomly to forward the query. Figure 2 illustrates a sample network scenario to understand the operation of flooding and random walk in a wired network. Let each vertex in the graph have link degree of 4 .

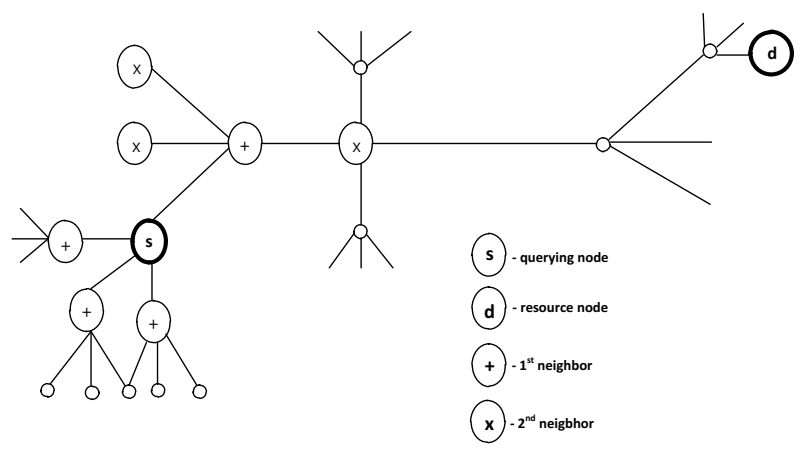

Fig.2. A sample network to demonstrate flooding and random walk strategies

Since it is a wired network scenario, the source and destination links are fixed. The flooding method will not scale well due to increase in the query messages generated at each hop which would be 4, 12, 36, so on respectively. If the resource is at a node located in the 3rd neighboring position from the resource requesting source node then the total message cost would be $4+12+36=52$ messages to resolve the query. The random walk method will generate less message cost, but 
consecutively more number of walkers will have to be set to randomly locate the resource. Only few vertices are visited even when the number of walkers is set at maximum, i.e., it will visit only 12 vertices of the $2^{\text {nd }}$ neighboring position even when 32 walkers are set. Hence the search is inefficient.

P2P networks due to their simple and distributed architecture are popular and one of the major stakeholders sharing internet traffic [1]. Several applications, like file sharing systems, multimedia services, database services, information retrieval systems, etc., are based on the peer-to-peer paradigm. These systems are broadly classified as structured and unstructured type. In structured P2P networks, the peers are well-organized and follow a strict structure based on complex algorithms. The famous examples being CAN, Chord, Pastry, Tapestry, etc. While the unstructured P2P networks do not require a predefined structure among the participating peers wherein nodes can join and leave arbitrarily. Among unstructured P2P protocols the most referred ones are Gnutella, Fastrack, Kazaa, Freenet, etc. A P2P in pure sense means that each peer has an equal role in the network. Even today, major mobile communication architectures are mainly centralized. One of the best possibilities to overcome the bottleneck due to the centralized architecture is to setup a P2P platform [2]. This communication pattern can suit any network including wired, wireless and even MANETs. Incorporating peer-to-peer network characteristics in MANET is coined as Mobile Peer-to-Peer (MP2P) network. In the past, $\mathrm{P} 2 \mathrm{P}$ resource discovery protocols have mainly focused on wired networks. But the performance results of these content searching methods cannot directly be compared with MANETs as there are many differences in the topology of both networks. Due to similarities in P2P and MANETs, a P2P overlay can run over mobile adhoc network. But at the same time their combination also poses difficulties due to differences in the operating layers, transmission mechanisms and rapid mobility in MANETs [3].

We initially start by analyzing the flooding, random walk and gossip based search methods under MP2P network. We study their performance under such dynamic network scenario. The flooding being a broadcast based technique has advantages over the other two unicast based methods under MANET [4]. We observe that mobility in MANET did not hamper the flooding based resource discovery process unlike the other compared ones. Even till date the most popular P2P file sharing application Gnutella is using flooding based scheme due to its simplicity and effectiveness [5]. To further lower the overhead in flooding technique we have to minimize the duplicate requests, network traffic and use the network and mobile resources effectively under MANET. In this paper we divide our work into two phases. First, we study neighbor discovery techniques for existing unstructured $\mathrm{P} 2 \mathrm{P}$ protocol and suggest an alternative way for P2P over MANET. Second, the enhancement of unstructured searching technique over MANET is proposed to lower the overhead. We present a density based query search mechanism to control flooding. We then extend our proposed method to adapt to the network dynamics efficiently considering the changing topology information from neighboring nodes to adjust the packet transmission for further improvement.
The rest of the paper is organized as follows. In section 2, we review related work on how resource discovery mechanism is carried out in P2P and MP2P network. Section 3 presents our proposed resource discovery protocols. Section 4 describes the simulation environment and the evaluated metrics. Section 5 shows the experimental results, and finally section 6 concludes with a summary.

\section{Literature Survey}

In this section, we start by discussing various broadcasting techniques in MANET. Then we provide a brief history of the past resource discovery techniques. In MANETs content discovery is related to routing protocols. In short, searching means routing a query to the node in the network. Broadcasting is a basic technique in MANET where one node sends a packet to all other nodes in the range. Broadcasting by flooding is a naive form of broadcasting in which every node retransmits the received packet once. But due to the random distribution of nodes in MANETs, this approach causes collision, redundancy and contention which leads to Broadcast storm [6]. To overcome this, in [7] a probabilistic approach for flooding was proposed wherein the rebroadcasting continues with a predetermined probability. Authors in [8] gives a brief comparison of various broadcasting techniques. They categorize the methods into simple flooding, probabilistic schemes, area based schemes and neighbor knowledge based schemes. They also provide a simulation based analysis of the compared techniques. Based on their observation in the probabilistic and area based schemes the rebroadcasting of nodes hampers the performance in congested networks.

Resource discovery in a pure $\mathrm{P} 2 \mathrm{P}$ system is to discover the IP address of the node that has the resource. Traditionally blind and informed search methods are used in unstructured P2P network. Among these, the flooding based techniques can cope up well with the churn and can readily locate the available objects. Many variants of flooding method were proposed such as modified BFS [9], teeming [10], expanding ring [11][12], blocking expanding ring search [13], etc. In expanding ring, the query is flooded with small TTL and then increases or even starts another flooding until the search succeeds. While the blocking expanding ring search is an extended version of the expanding ring in which the new round of search starts with a higher TTL value from where the last attempt was terminated. In [14] a controlled flooding based search strategy for unstructured P2P network is proposed. In their work the probability of forwarding the query to the selected neighbor is based on the number of nodes connected at the overlay layer and the distance information is calculated between the query initiator node and the other nodes. A hybrid flooding scheme was suggested in [15]. Their work is based on the selection of nosey nodes to forward the query request. They initially started with the flooding scheme for some limited number of hops and then proceed to forward the query to the node which has maximum number of connections as the neighbor, i.e., the nosey nodes. But their search design is based on these nosey nodes that act as super peers. Alternative search schemes were also proposed that are a mixture of 
both probabilistic and deterministic types. One such method was proposed which resembled flooding with independent k-walkers. Starting with the flooding approach, the search is extended to k-walkers until query is resolved. In [16][17], an approach with a combination of simple and tree-based flooding was proposed. But this had high tradeoff in terms of cost. To overcome this authors in [18] proposed a dynamic search scheme that takes advantage of flooding and random walk approaches based on the context of the search. Adaptive Probabilistic Search (APS) scheme builds knowledge on the basis of the past experience. An improvement of APS was proposed in [19]. They used the Ant colony optimization that considers the file type and relevant score obtained by past history information.

Most of the above discussed works are evaluated over wired and fixed scenarios. Self organizing networks such as MANET and $\mathrm{P} 2 \mathrm{P}$ have many limitations and challenges like maintaining continuous network connectivity, frequent disconnection, limitations of bandwidth, churn, etc. Directly applying P2P characteristics over MANETs may not be the ideal choice. In [20] they suggest an architectural approach to suit well for MP2P network around multicast communication. Authors in [21] propose a multicast based P2P content distribution protocol for MANETs. Their protocol sticks to the MANET characteristics. Even in most P2P file sharing protocols over MANET the file search resembles the flooding technique.

Like in ORION [22], it combines Gnutella-like flooding technique with AODV routing protocol. While in MPP [23] and EDSR [1] for file search, it combines Gnutella-like flooding technique with DSR routing scheme. Many resource discovery protocols proposed for Mobile Peer-to-Peer networks also uses flooding model as the basic search mechanism. In [24] a selfaware search protocol based on the query-flooding approach is proposed. Here they initiate a flood of requests to all neighbor peers and then based on the positive reply, ranking of the nodes is done. Further such nodes that have a common subject of interest form a group. For every such group a super node is associated which is responsible for communication between different groups. In [25] a resource discovery algorithm based on super node for MP2P is presented. The basic idea of their work is to choose the nodes with high capacity and weak dynamics as super node to transmit the query further. Here also flooding model is used to forward the query among other super nodes. The protocols intended for wired networks have poor performance in such dynamic environment [26].

The efficiency of $\mathrm{P} 2 \mathrm{P}$ content discovery protocol on MANET have been evaluated in [27]. They suggest that unstructured protocols work better than the structured one over MANET. In [28] author show that mobility and mobility models have a strong affect on the performance of P2P protocols over MANET. For a Mobile P2P network due to mobility it further rules out the possibility of applying knowledge based schemes as the topology changes rapidly and such past available information may not be feasible at the very next instance. While the blind searching methods will increase the query message cost incurred due to duplication of query message and also consume extra battery power and network bandwidth in the process. In the above discussed works, node mobility hampers the search efficiency thereby increasing the network overhead and query delay. Therefore, new resource searching algorithms based on characteristics of Mobile P2P networks have to be focused on.

To overcome the above issues, in this article we suggest an optimal density based resource discovery protocol that considers the local topology information to improve the search efficiency while eliminating the duplicates that hinders the search process. Furthermore, an extension to the density model is proposed that also uses neighboring node's density information with local node density information to further improvise the search. We also noticed that mostly all the resource search techniques proposed for Mobile Peer-to-Peer network were mainly based around super peer architecture, i.e., it utilized super nodes to process the query. Here we focus on the fully decentralized pure P2P network architecture where our proposed resource discovery scheme is not bound around any special node, i.e., for a flat topology that works without any hierarchy information.

\section{Flooding-based Resource Discovery Algorithms}

Generally neighbor nodes are discovery by doing a 1-hop broadcast hello protocol. To establish a connection with existing nodes, the newly arrived node broadcast a HELLO message within its 1-hop transmission range. The other nodes upon receiving the HELLO message reply with a HELLO RESPONSE message. In this way, the neighbor list is updated frequently in the overlay. But doing it the traditional way will again increase the broadcast overhead. So we use the neighbor information which is available with the routing protocol and pass this information to the $\mathrm{P} 2 \mathrm{P}$ application. We maintain a neighbor list and a neighbor count resolved from the routing layer neighbor list information. The algorithm given in Table 1 explains the way node count is maintained for a P2P application running over MANET.

Table 1 Neighbor count algorithm

\begin{tabular}{l}
\hline Algorithm 1: Node Count ( ) \\
\hline if (strcmp(argv[1], "neighbor_count")==0) then \\
1. Initialize nbrs $=0$ \\
2. Point to the first element in neighbor cache from \\
3. AODV_Neighbor \\
4. Increment and update nbrs. \\
5. Pass this information to the P2P application. \\
end if
\end{tabular}

We model various unstructured $\mathrm{P} 2 \mathrm{P}$ resource discovery algorithms under MANET. Here, the meaning of resource discovery for a standard $\mathrm{P} 2 \mathrm{P}$ system is nothing but discovering the address of the node in the network which actually has the requested resource. So from the resource reply which will be sent by a resource containing node, the querying node will resolve the address of the resource provider.

\subsection{Simple Flooding Scheme}

In this method, the query initiator node sends the resource request message over a 1-hop broadcast. All the 1-hop neighbors receiving that message in turn rebroadcast the message with the fixed transmission nrobabilitv the first 
time it receives the message. Duplicated requests will be dropped. If any neighbor has the resource, then the query will be resolved. But even after the resource reply, all other nodes will continue forwarding the message until reaching all the nodes in the network. The message is flooded throughout the network. Here the transmission probability (TP) is fixed, i.e., $100 \%$. But this method causes high network traffic and results in a broadcast storm. The following algorithm explains the flooding scheme as given in Table 2 .

Table 2 Simple flooding algorithm

Algorithm 2: Simple flooding $(\mathrm{m})$

1. upon reception of message $m$ at node $n$

2 . if message $m$ received for the first time then

3. $\operatorname{broadcast}(\mathrm{m})$

\{all nodes within the range receive the message 4. end if

\subsection{Probabilistic Flooding Scheme}

This method is similar to flooding except that here the nodes rebroadcast the message with a predetermined transmission probability. The querying node sends the resource request message over a 1 -hop broadcast. All the 1-hop neighbors on receiving that message rebroadcast it with a pre-assumed probability. Here the message overhead is reduced as compared to simple flooding, but this scheme has poor coverage reach due to its uniform pattern where every node rebroadcasts with the same transmission probability irrespective of the topology. So in a situation where only few nodes are connected to each other then nodes may not receive the broadcast packet unless TP is high enough. The following algorithm explains the probabilistic flooding scheme as given in Table 3.

Table 3 Probabilistic flooding algorithm

Algorithm 3: probabilistic flooding(m,TP)

1. upon reception of message $m$ at node $n$

2 . if message $m$ received for the first time then

3 . broadcast $(\mathrm{m})$ with transmission probability $T P$

\{all nodes within the range receive the message

4. end if

\subsection{Density based Flooding Resource Discovery Scheme (DiFlooding)}

Due to the random distribution of nodes in MANETs, the probabilistic flooding scheme has an unpredictable behavior with the predetermined transmission probability. In a dense region where the number of nodes is high, the preset TP may unnecessarily utilize the network resources and drain the battery power due to retransmission of the message which is mostly not needed as many nodes overlap along the same transmission range. While in a sparse region, the preset value may not scale well and in most cases the nodes may not receive the query request. So there is a need for varying the TP as per the local node density in such dynamic network scenario. In this method, the node's density is considered to vary the transmission probability. On receiving a broadcast resource request packet at node $X$, it gets the neighbor count of the node, i.e., number of neighbors from AODV routing agent of the node. Based on this information, the $\mathrm{TP}$ is set accordingly. We propose an Adjusted Transmission Probability Algorithm (ATPA) based on the density of the node as given in Table 4. For experiments, the values of the threshold MinNeigbhors and MaxNeigbhors are set as 3 and 20 respectively.

Table 4 Adjusted transmission probability algorithm

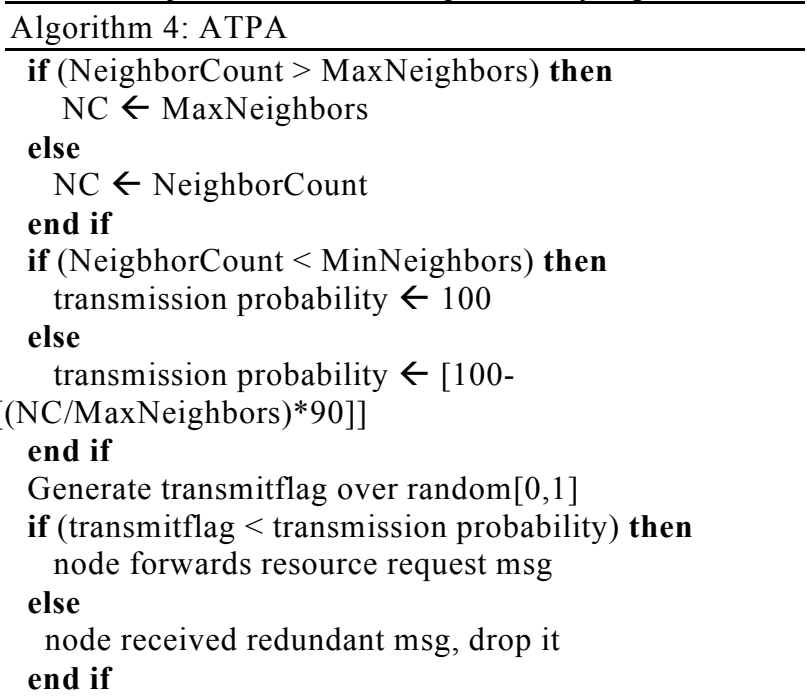

In our method, the broadcast storm issue is reduced. The following algorithm explains the proposed density based optimal flooding resource discovery scheme as shown in Table 5.

Table 5 Density based flooding algorithm

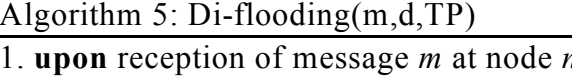

2 . if message $m$ received for the first time then

3. get the current node density $d$

4. broadcast $(\mathrm{m})$ with transmission probability $T P$ as per density $d$

\{all nodes within the range receive the message 5. end if

3.4 Neighbor's Density Based Local Flooding Resource Discovery Scheme (NDiFlooding)

In our previous approach, the resource discovery request will be broadcasted based on the current node density. In highly dynamic MANETs the topology changes very frequently, considering node density will be useful but to boost the process considering density information at very previous instance will also be helpful. If the set of nodes have only few intermediate nodes then there is a chance that message is not forwarded due to failure.

While if there is a dense cluster of nodes then there will be chance of collision and redundancy. To further reduce the effect of redundant messages and lower network overhead, we propose a neighbor's density based model to dynamically adapt TP to suit such mobile network. In this method, we also get the neighbor density information from where the resource request message was forwarded from the previous hop. Then the node will forward the message based on knowledge of both the local node neighbor density and the previous node's neighbor density information, i.e., density history of the neighbor node along with the current node density 
information is used appropriately to vary the TP. Doing so each node will adaptively decide to forward or drop the received request based on this information. The main idea here is to efficiently adapt to the network dynamics to minimize failures and collisions. In this scheme, if the past neighbor's density is more than the current local node density then there is a need to give high priority by increasing the TP relatively. But if vice versa, then there is need to give low priority by decreasing the TP in proportion to the corresponding neighborhood information. So here unnecessary re-broadcasting is avoided and collision is reduced saving the network and mobile resources, while for loosely connected nodes the $\mathrm{TP}$ is set accordingly to avoid failures by also considering the neighboring node density information. The following algorithm explains the neighbor's density based local flooding scheme as given in Table 6 .

Table 6 Neighbor's density based flooding algorithm

Algorithm 6: NDi-flooding(m,d1,d2,TP)

1. upon reception of message $m$ at node $n$

2 . if message $m$ received for the first time then

3 . get previous node's neighbor density $d 1$

4. get current node's neighbor density $d 2$

5 . broadcast $(m)$ with transmission

probability $T P$ as per densities $d 1$ and $d 2$

\{all nodes within the range receive the message

6 . end if

\section{Simulation Evaluation}

In this section, we evaluate the performance of the various resource discovery algorithms. We simulate a P2P application over MANET and follow a P2P resource discovery scenario. For each protocol and network size, 3 different scenarios are created, and an average analysis is done to evaluate the results.

Our proposed methods will be compared with the simple flooding and the probabilistic flooding scheme. The parameters of the simulation are as follows:

\section{Common Parameters}

$\begin{array}{ll}\text { Channel } & \text { WirelessChannel } \\ \text { Propagation Model } & \text { TwoRayGround } \\ \text { Physical Model } & \text { WirelessPhy } \\ \text { Mac Protocol } & 802 \_11 \\ \text { Queue Type } & \text { DropTail PriQueue } \\ \text { Queue Length } & 50 \\ \text { Antenna Type } & \text { OmniAntenna } \\ \text { Energy Model } & \text { Battery } \\ \text { Mobility Model } & \text { Random way point } \\ \text { Initial Node Energy } & \text { 500 Joules } \\ \text { Routing Protocol } & \\ \text { P2P RD Protocol } & \text { Flooding, ProbFlooding, } \\ & \text { DiFlooding, NDiFlooding } \\ \text { No of Queries } & 1-10 \\ \text { Simulation Time } & 120 \mathrm{sec} \\ \text { Query Interval } & 5 \mathrm{sec}\end{array}$

Nodes and Network scenario

Number of Nodes

Resource Providing Nodes
Topography Size

$1000 \mathrm{~m}$ $\mathrm{x}$

$1000 \mathrm{~m}$

Pause Time

$20.00 \mathrm{sec}$

Node speed

$20.00 \mathrm{~m} / \mathrm{s}$

\section{P2P Application Parameters}

P2PApplicationPort 6346

P2PResourceRequestMessageSize

P2PresourceReplyMessageSize

Broadcast Delay

Transmission Probability

$100 \%$

100 bytes
$0.01 \mathrm{sec}$ bytes
Simple flooding -
Probabilistic flooding
$-50 \%$

\subsection{Performance Metrics}

The analysis and performance measurement of the resource discovery algorithms are based on the following metrics, as shown in Fig 3.

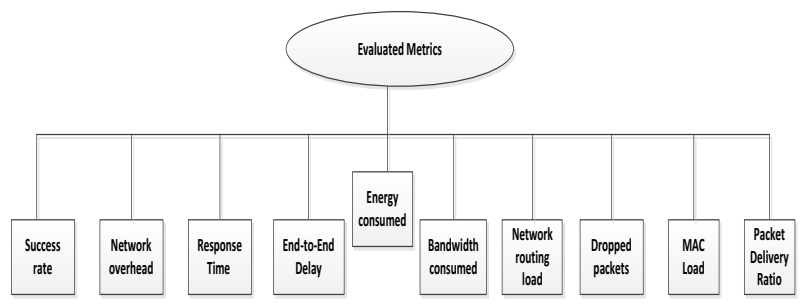

Fig.3. Performance measures

We present the definition of metrics as follows:

\section{a) Success Rate}

The success rate is an important metric for measuring the performance of $\mathrm{P} 2 \mathrm{P}$ resource discovery algorithm. It is a ratio of total generated requests and the successfully received replies. It is measured as follows:

Success Rate $=\left(\mathrm{R}_{\mathrm{ep}} / \mathrm{R}_{\mathrm{eq}}\right) * 100 \quad(\%)$

where

$\mathrm{R}_{\mathrm{eq}}$ is the total generated resource requests and

$R_{e p}$ is the total successfully received resource replies

The higher success rate signifies the better performance of the resource discovery algorithm.

\section{b) Avg. Resource Discovery Time}

The resource discovery time is also another important metric for measuring the performance of $\mathrm{P} 2 \mathrm{P}$ resource discovery algorithm. It is the time difference between the resource request generation time and the resource reply received time. It is calculated as follows:

\section{Resource Discovery Time $=\mathrm{T}_{\text {Rep }}-\mathrm{T}_{\mathrm{Req}}(\mathrm{sec})$}

The lower response time signifies the better performance of the resource discovery algorithm.

\section{c) P2P Network Overhead}

In this work we measure Overhead in terms of total number of generated and forwarded routing messages at the Network layer. 


\section{d) Avg. Consumed Energy of P2P Network}

Generally the consumed energy is calculated as the average energy consumed by all the nodes of the network for the entire time of the simulation. It is measured in Joules. It is calculated as follows:

$$
E_{\text {Avg }}=\frac{1}{N} \sum_{i=1}^{N} I E_{i}-F E_{i}
$$

(Joules)

where

$$
E_{A v g} \text { is the calculated Avg. Consumed Energy of }
$$
P2P network in Joules

$\mathrm{N}$ is the total number of nodes in the P2P network

$\mathrm{IE}_{\mathrm{i}}$ is the initial battery Energy of the Node I

$\mathrm{FE}_{\mathrm{i}}$ is the final battery Energy of the Node I

\section{e) P2P Agent Level Throughput (kbps)}

Normalized throughput is a measure of number of messages or data packets successfully delivered over time. It is generally measured in Kbps or Mbps. Here P2P Agent Level Throughput is calculated as follows:

$$
\text { Throughput }=\mathrm{A}_{\mathrm{rs}} / \mathrm{T}_{\mathrm{d}} \quad \text { (kbps) }
$$

where

$$
\begin{aligned}
& \mathrm{A}_{\mathrm{rs}} \text { is the sum of all the packet sizes received at } \\
& \text { the P2P Agent Level of all the nodes in the } \\
& \text { network. } \\
& \mathrm{T}_{\mathrm{d}} \text { is the total time duration of the P2P resource } \\
& \text { discovery scenario }
\end{aligned}
$$

\section{f) Overall Dropped Packets}

A dropped packet in a MANET scenario is also an important metric to evaluate the network performance. Generally packets will be dropped due to several reasons in a wireless adhoc network scenario. And particularly this dropping of packets in a typical P2P network is expected to be high since these algorithms will use lot of duplicates of the same resource discovery message during the process and that will increase the overhead causing network bottleneck. We count the packets dropped at all the network layers of all the P2P nodes.

\section{g) P2P Network MAC Load}

Generally MAC load is calculated as a ratio between the number of packets sent at MAC layer and the number of data packets successfully delivered. But here we calculated MAC load as a ratio between the number of packets sent at MAC layer and the number of resource requests received at P2P Agent level of each node. Here P2P Network MAC Load is calculated as follows:

$$
\text { P2P Network MAC Load }=M_{s} / A_{r}
$$

where

$\mathrm{M}_{\mathrm{s}}$ is the total MAC layer generated or sent packets

$\mathrm{A}_{\mathrm{r}}$ is the total P2P agent level received packets

\section{h) P2P Network Routing Load}

Generally normalized routing load is a ratio between the number of routing packets generated and the number of data packets successfully delivered. But in this P2P network scenario we measure it differently. Here routing load is measured as a ratio between the number of routing packets generated + forwarded and the number of resource discovery messages received at application layer of each node in network during the resource discovery process. It is calculated as follows:

$$
\text { Routing Load }=\left(R_{g}+R_{f}\right) / A_{r}
$$

where

$$
\begin{aligned}
& \mathrm{R}_{\mathrm{g}} \text { is the total generated/sent routing packet } \\
& \mathrm{R}_{\mathrm{f}} \text { is the total forwarded routing packets and } \\
& \mathrm{A}_{\mathrm{r}} \text { is the total } \mathrm{P} 2 \mathrm{P} \text { agent level received packets }
\end{aligned}
$$

The lower value signifies the better performance of the resource discovery algorithm.

\section{i) P2P Agent Level Hop to Hop Packet Delay}

This metric is a refined version of normal delay calculation. Here instead of calculating delay between source and destination, it is calculated for each packets generated during each of the protocol in process i.e. it is the time taken for a packet to reach each hop during forwarding the resource discovery request. It is calculated as follows: Where

Hop to Hop Packet Delay $=\mathrm{T}_{\mathrm{r}}-\mathrm{T}_{\mathrm{f}}(\mathrm{ms})$

$\mathrm{T}_{\mathrm{r}}$ is the time at which a node receives a resource discovery request and

$\mathrm{T}_{\mathrm{f}}$ is the time at which that resource discovery request was forwarded from the previous hop.

Minimum delay signifies the better performance of the resource discovery algorithm.

\section{j) P2P Agent Level Packet Delivery Ratio (PDF)}

Here we measure PDF as a ratio of total sent messages at the $\mathrm{P} 2 \mathrm{P}$ agent and the total messages received at the $\mathrm{P} 2 \mathrm{P}$ agent (it means that we are not just counting the successful resource replies only as the received packet count)

$$
\mathrm{P} 2 \mathrm{P} \text { Agent Level PDF }=\left(\mathrm{A}_{\mathrm{r}} / \mathrm{A}_{\mathrm{s}}\right) * 100
$$

where

$A_{r}$ is the count of all the packets received at the P2P Agent Level of all the nodes in the network.

$\mathrm{A}_{\mathrm{s}}$ is the count of all the packets sent at the P2P Agent Level of all the nodes in the network.

Generally in MANET, the PDF value of 100 signifies the highest performance. But here we may get more than 100 because of the broadcast technique where the received packet count will be much higher than the sent packet count. So interpretation of PDF in this case is entirely different from the normal MANET.

\section{Results}

\subsection{Evaluating performance metrics}

To check the robustness of the simple flooding and our proposed methods under highly dynamic mobile network scenario we evaluated the resource discovery protocols 
and verified them for each metrics. Fig.4 shows the comparative performance of the resource discovery algorithms in terms of success rate. As seen in the graph, our proposed schemes are almost equal to flooding. While the probabilistic scheme also shows good results. Success rate of flooding is almost $100 \%$ while for our schemes the success rate is just marginally low around 95 to $97 \%$. The probabilistic flooding technique also has success rate above $85 \%$. Flooding provide successful resource discovery because, even though the moving request at one node fail, the same copy of the request will be forwarded from another node, as each node rebroadcasts all the packets so it will have a high possibility of reaching the destination.

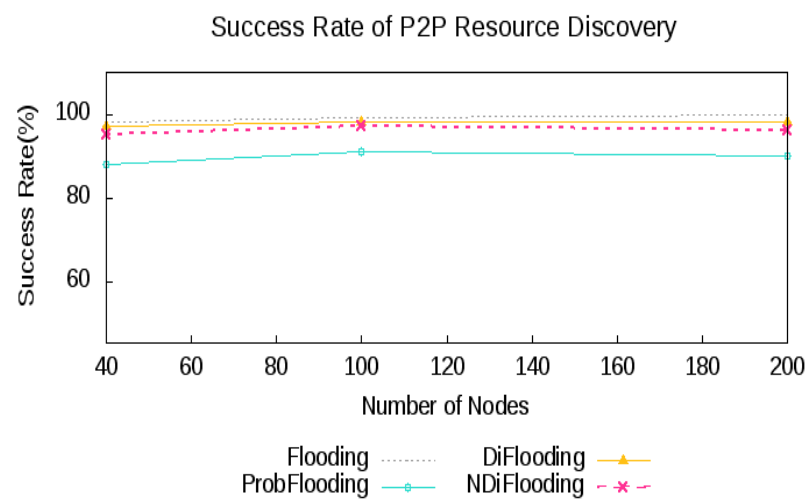

Fig.4. Comparisons of success rate

Fig.5 shows the query response time for the various resource discovery schemes. Our techniques has the least request delay compared to the other methods. Our schemes give quicker response because of the appropriate variation of the TP using the local density information and intermediate neighborhood information. While in flooding the resource reply may get lost most times.

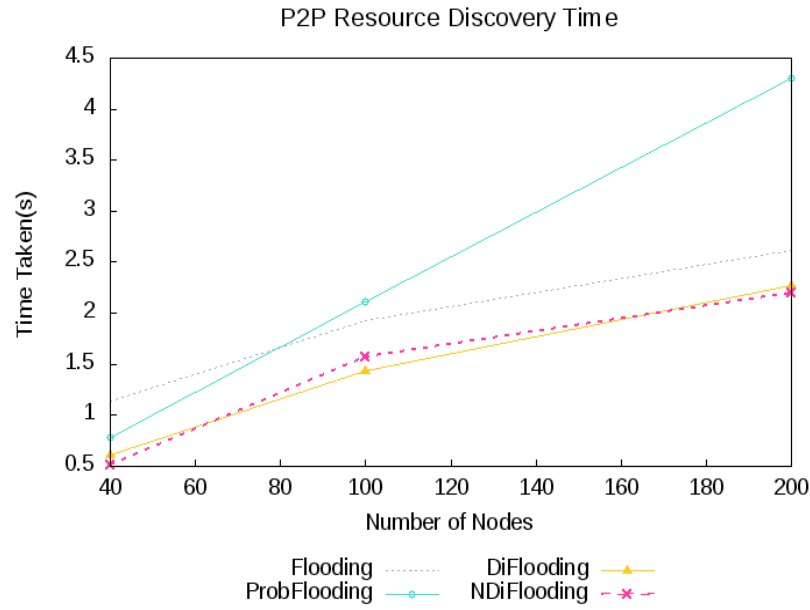

Fig.5. Comparisons of query response time

Fig.6 shows the generated overhead for the various resource discovery algorithms. The proposed schemes has very low overhead than the other compared algorithms as unnecessary re-broadcasting is avoided thereby reducing the redundancy.

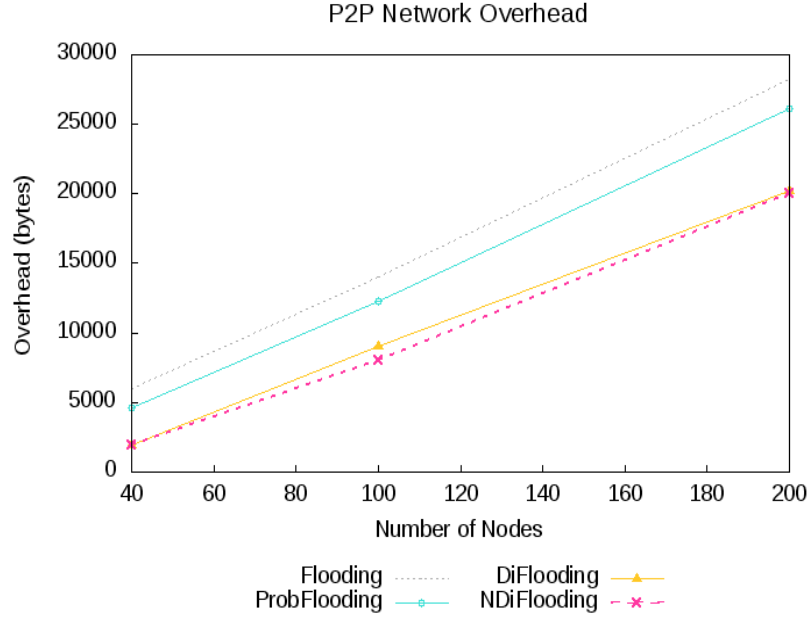

Fig.6. Comparisons of overhead

Fig.7 shows the performance of various searching techniques in terms of energy consumed. Our proposed technique lowers the battery power consumption which is an important parameter with respect to mobile nodes. Our methods consume less power since not all the nodes are involved in retransmission as the broadcast is based on the mixed knowledge of current and neighborhood density information.

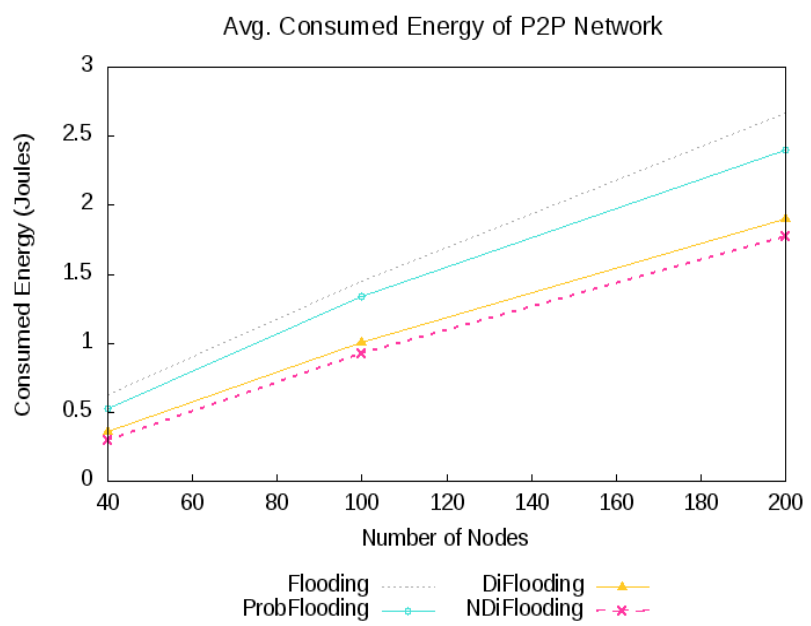

Fig.7. Comparisons of battery power consumed

A good resource discovery protocol consumes less bandwidth (i.e., less throughput) and also should provide equally good success rate. Fig.8 shows comparisons of throughput for various protocols. Our proposed methods are the best among the other compared ones.

Fig.9 shows the performance of the resource discovery protocols in terms of dropped packet count. As seen in the graph, the proposed techniques have lower failure rates. At a dense region there will be high chance of packet collision. The proposed methods vary its transmission probability at that point giving low priority based on the local topology knowledge as well as based on both the local and neighboring node information which will reduce packet loss. 
P2P Agent Level Throughput

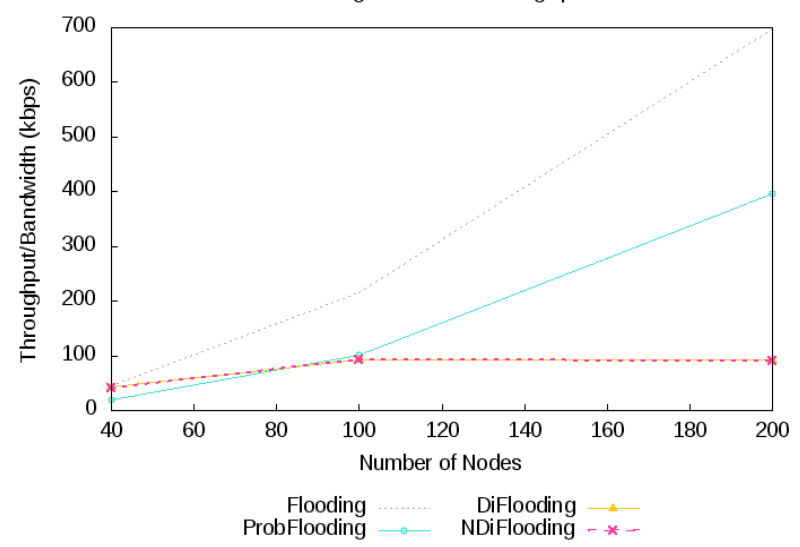

Fig.8. Comparisons of throughput

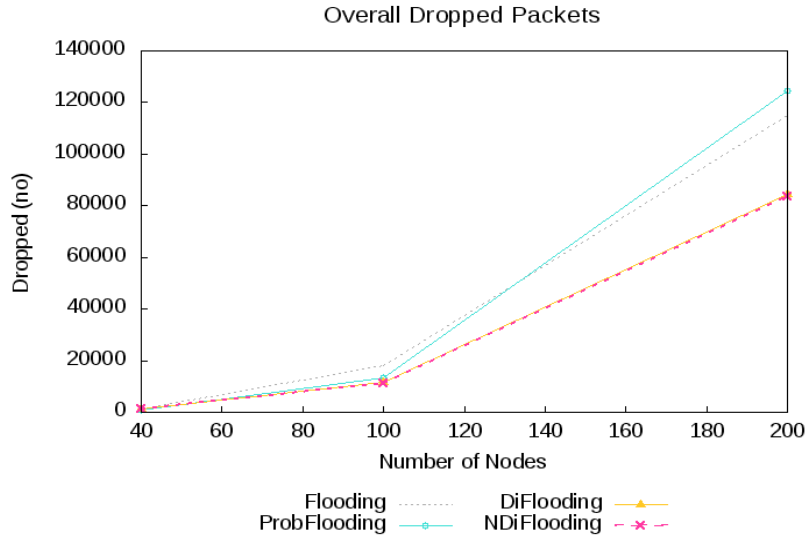

Fig.9. Comparisons of dropped packets

Fig.10 shows the comparative performance of the resource discovery algorithms in terms of MAC load. Our proposed methods have very low MAC layer overhead then the compared methods. In our methods the node will vary transmission probability based on the current node density and also the neighboring node's density thereby restricting the involvement of all nodes in broadcasting.

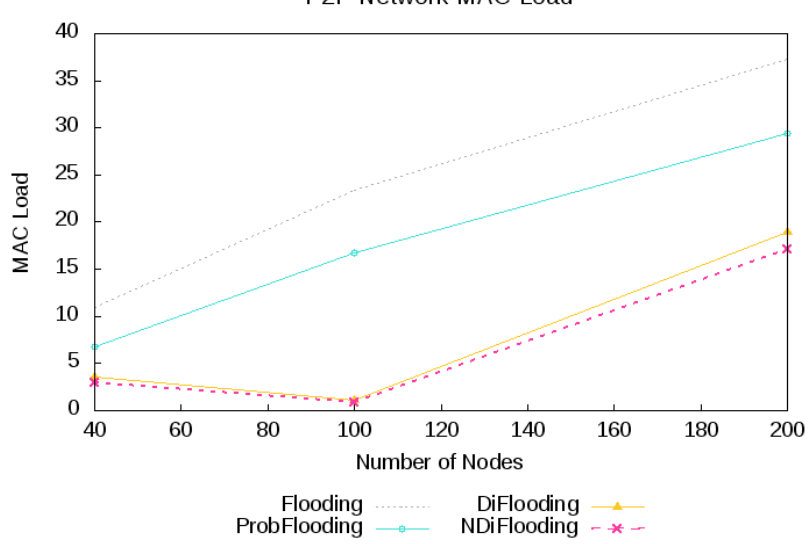

Fig.10. Comparisons of MAC load

As seen in Fig.11 the proposed technique has very low routing load than the compared methods.

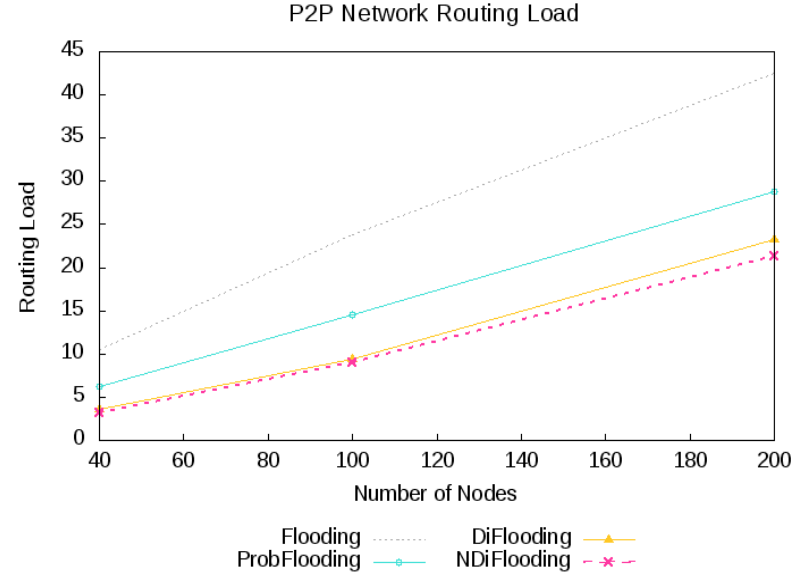

Fig.11. Comparisons of routing load

Fig.12 shows the performance of the resource discovery protocols in terms of hop to hop delay. The flooding technique shows better performance, while our proposed techniques also have almost equal performance initially.

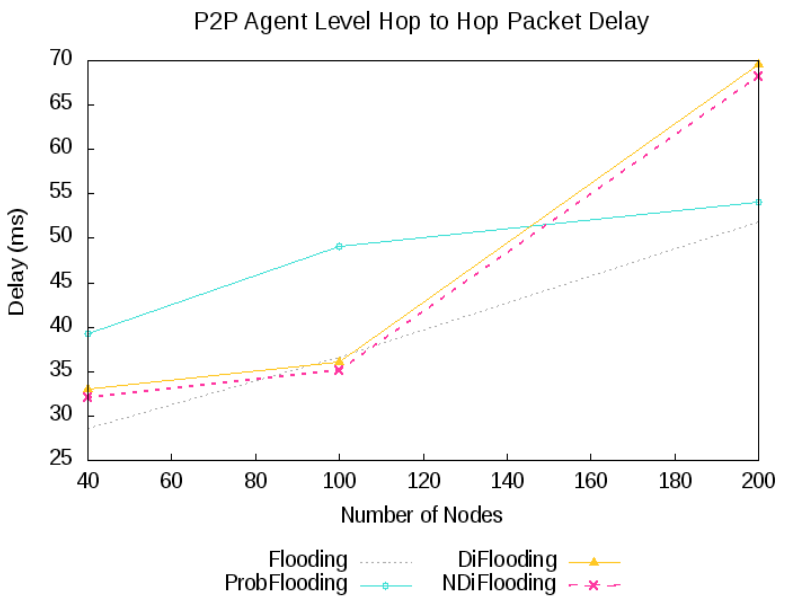

Fig.12. Comparisons of hop to hop delay

In Fig.13, the proposed algorithms show better performance as compared to the flooding method in terms of packet delivery ratio. The probabilistic flooding scheme outperforms the other techniques marginally.

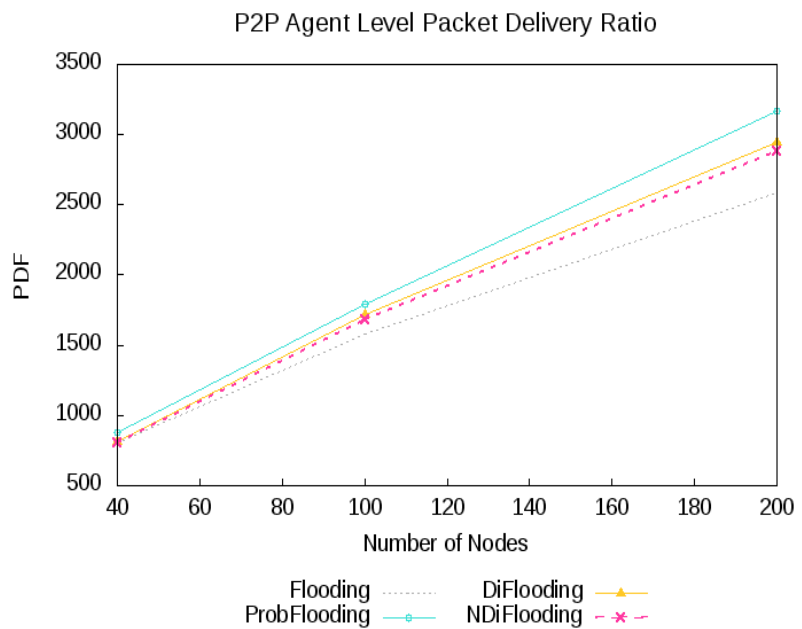

Fig.13. Comparisons of hop to hop packet delivery ratio 
From the above results we observe that our proposed schemes has low routing overhead as to the compared techniques. Further our method is crucial in such constraint oriented environment where the battery power and network resources are very critical. Our density based schemes minimize the power consumption, reduce search latency, lower bandwidth consumption and yet provides equally good success rate.

\subsection{Varying Node Mobility}

To evaluate the robustness of our proposed DiFlooding scheme under highly dynamic mobile network scenario we evaluate the resource discovery protocols under different node speeds, i.e., $10 \mathrm{~m} / \mathrm{s}, 30 \mathrm{~m} / \mathrm{s}, 50 \mathrm{~m} / \mathrm{s}$ and $70 \mathrm{~m} / \mathrm{s}$, respectively, and verify all important metrics like search efficiency, energy consumed, overhead and response time. We study in two different network sizes of 40 and 100 nodes, respectively. Fig.14 and Fig.15 show the overhead generated for different mobility. Our proposed technique has lower overhead as compared to the flooding method.

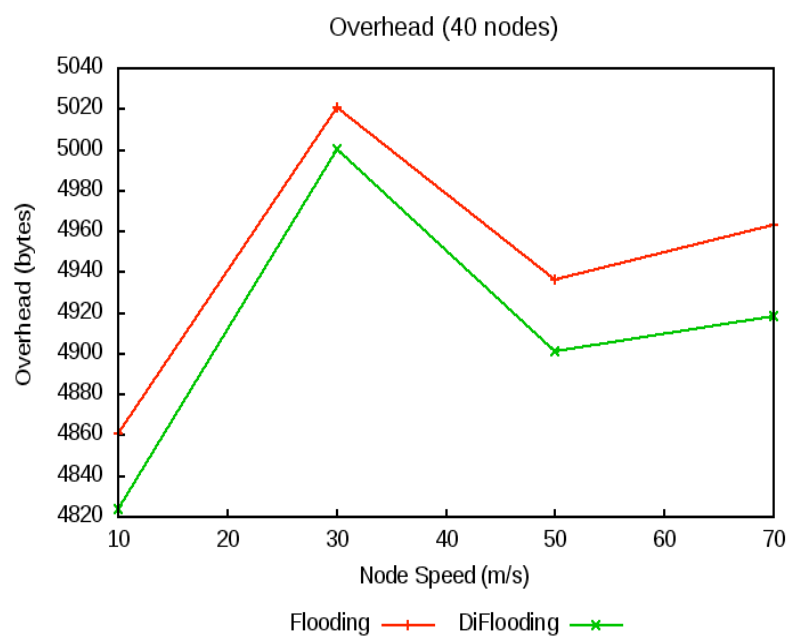

Fig.14. Comparisons of generated overhead

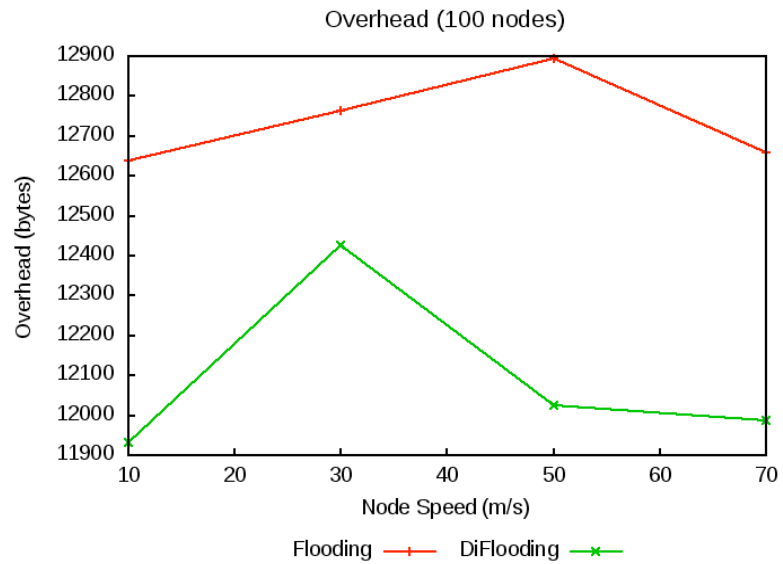

Fig.15. Comparisons of generated overhead

Fig.16 and Fig.17 show the battery power consumed by flooding and density based scheme under varying node speed. Our proposed method consumes lower power as compared to the flooding technique.

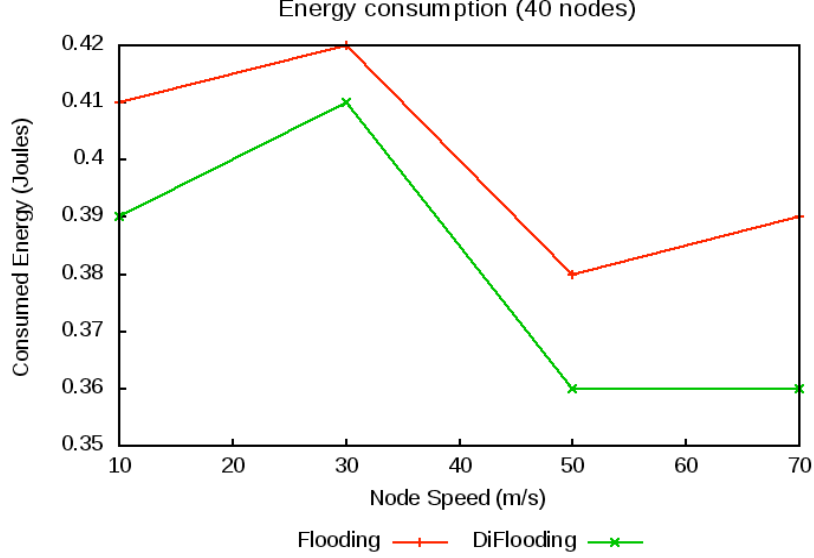

Fig.16. Comparisons of energy consumed

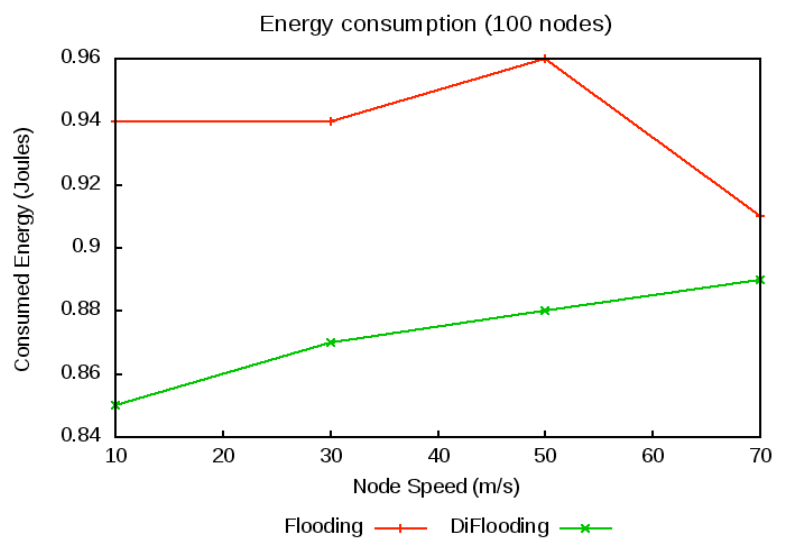

Fig.17. Comparisons of energy consumed

Fig.18 and Fig.19 shows the query delay comparison between both the methods against different node mobility. Our proposed method shows good results by minimizes the query delay as to the compared method.

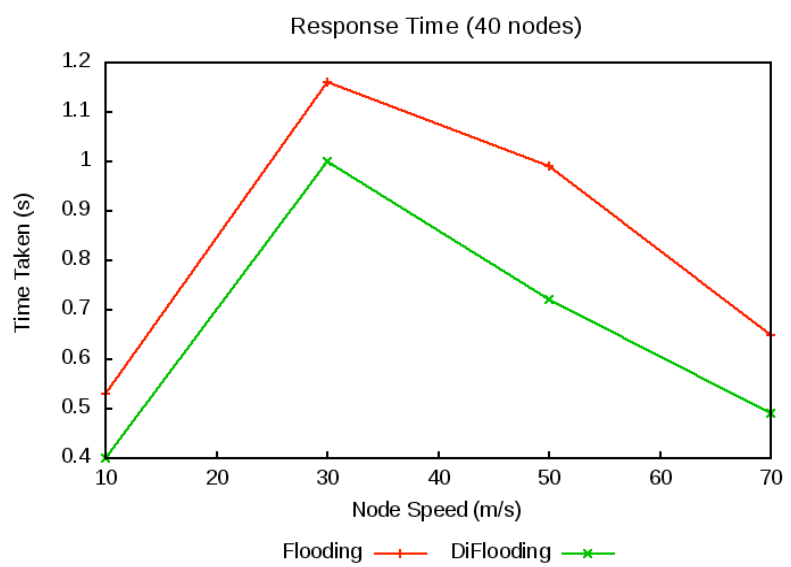

Fig.18. Comparisons of response time 


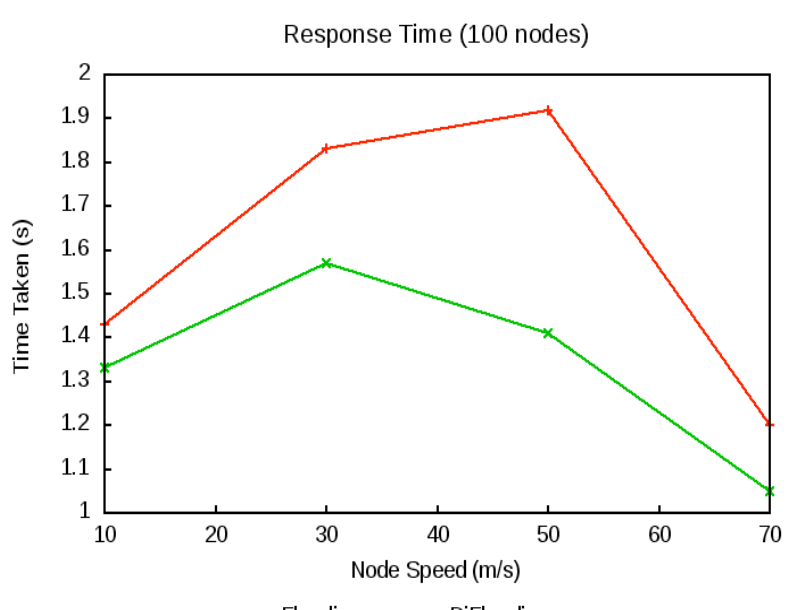

Fig.19. Comparisons of response time

In Fig.20 and Fig.21, we compare the efficiency of the proposed method with the flooding scheme. From the results it is observed that mobility will not affect resource discovery under flooding. Our proposed method initially has equal efficiency but with increase in mobility the performance reduces a bit.

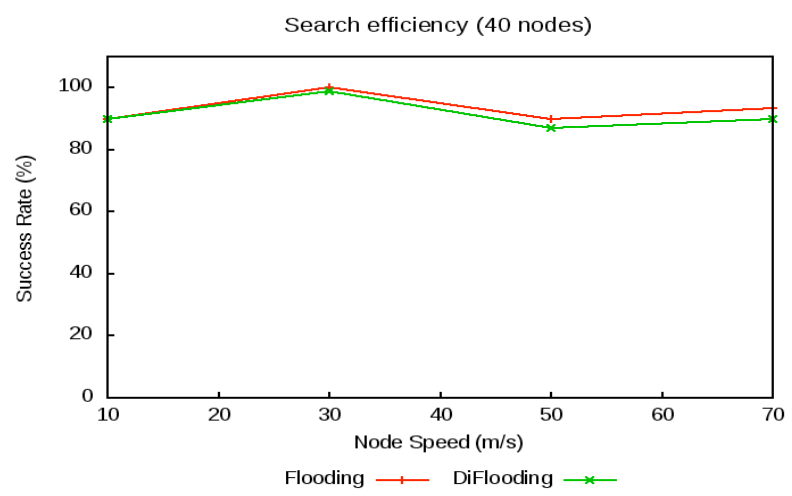

Fig.20. Search efficiency

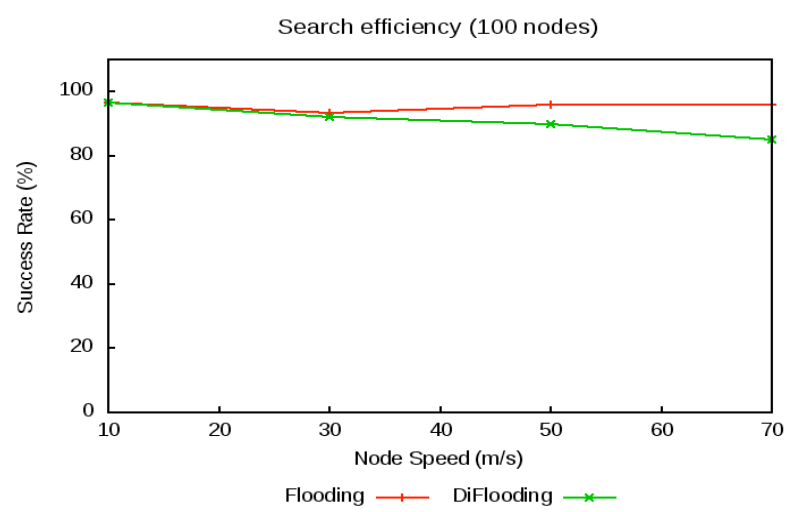

Fig.21. Search efficiency

We further analyze the behavior of the resource discovery schemes for two different network categories

1) Less Dynamic [1-9 node speed]

2) Highly Dynamic [10-70 node speed]

To study, the robustness of flooding and our proposed density based scheme under highly dynamic mobile network scenario we evaluate the resource discovery protocols for a sample of 40 and 100 nodes, respectively.

In Fig.22, we evaluate the performance in terms of success rate for the different network categories. The first pair of results seen in the graph is for 40 nodes while the second pair results is for 100 nodes. As seen in the graph, the flooding technique has good performance in both environments. Our method shows good stable performance under less dynamic network scenario but it marginally lowers under high dynamics.

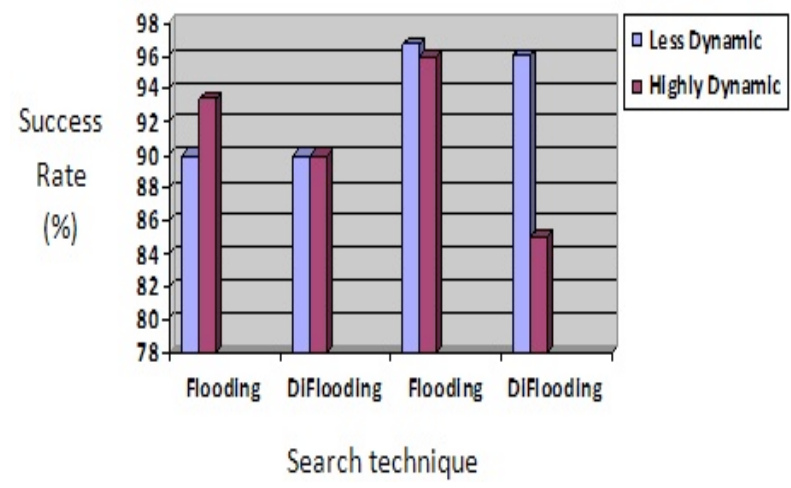

Fig.22. Comparisons of query success rate

Fig.23 shows the comparison of search overhead under different environments for flooding and the density based scheme. Our proposed scheme has lower overhead for both the network scenarios.

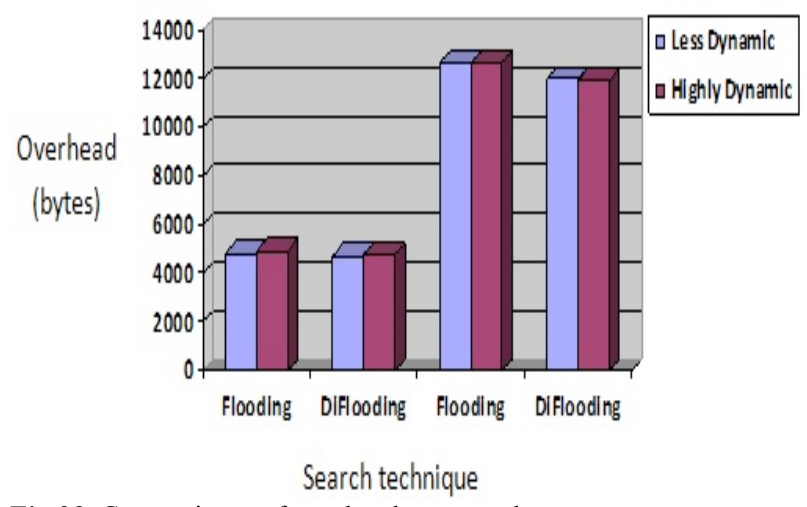

Fig.23. Comparisons of overhead generated

Saving the resources especially battery power is critical with mobile nodes. In Fig. 24 we evaluate the performance in terms of overall energy consumed in both the techniques. Our proposed density based method consumes less power than the simple flooding which is crucial in mobile network.

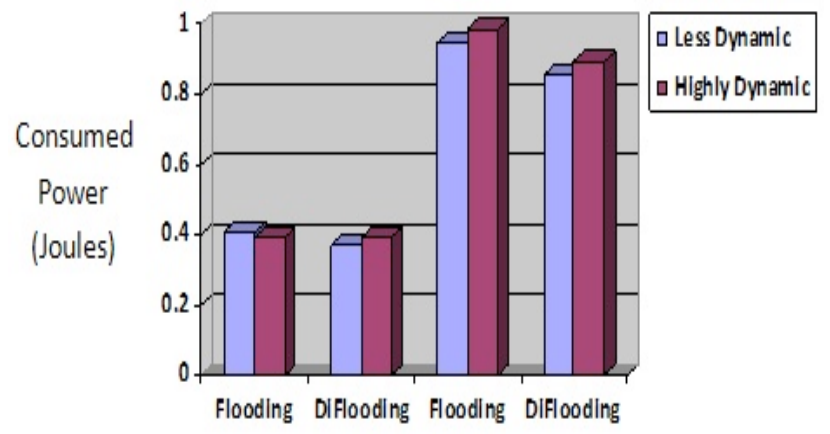

Search technique 
Fig.25 shows the performance of the compared techniques in terms of query response time under the different network environment. As seen in the graph, our proposed method minimizes the query delay under both the network scenarios than the simple flooding technique.

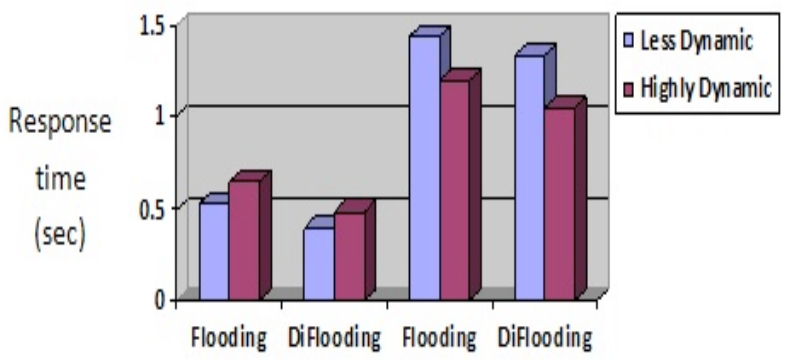

Search technique

Fig.25. Comparisons of query delay

We verify from the above results that our basic density model is less hampered by the effect of varying node speed as compared to flooding technique. The reason for this is the limiting of unnecessary rebroadcasting as compared to flooding thereby reducing the collision, redundancy and contention. So overall our method reduces the overhead, minimizes the query delay and saves the battery power due to minimum retransmission.

\subsection{Different Query Request Mode and Resource Replication Scenarios}

We also evaluate the Flooding and DiFlooding method to verify the performance under periodic and concurrent query requesting scenarios. In the periodic request, the node fires the query after every interval of $5 \mathrm{sec}$. While in the concurrent query request scenario, the nodes fire the query simultaneously.

Here the following questions must be investigated.

1. What is the effect of each scenario on the network overhead?

2. Effect on the response time, search efficiency and the energy consumed by the nodes?

Fig. 26, Fig.27, Fig.28 and Fig.29 show the generated overhead, energy consumed, query response time and success rate between the proposed and the flooding search technique for a sample of 80 nodes for different query requesting scenarios. From the graphs it is observed that our proposed density based method is a good alternative to the compared technique as it lowers the overhead, battery power and response time while providing almost equal success rate.
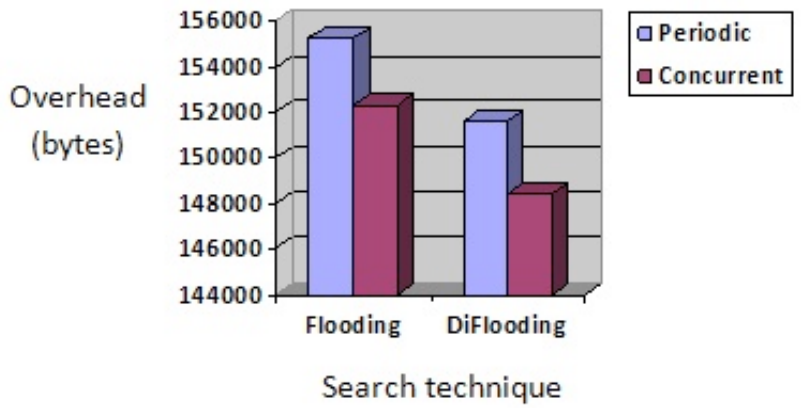

Fig.26. Overhead for different request type

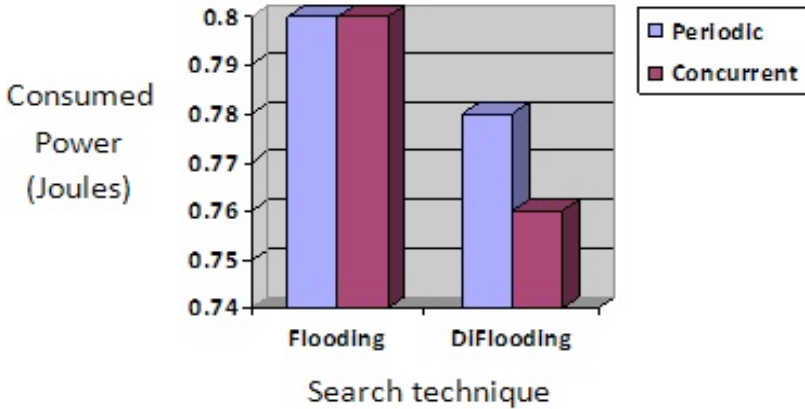

Fig.27. Consumed power for different request type
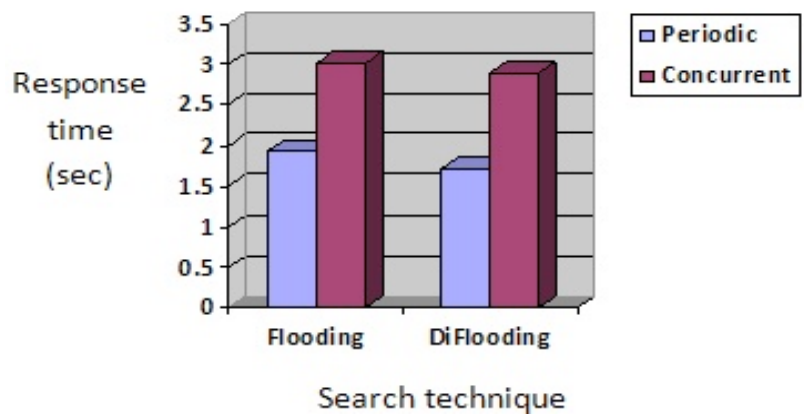

Fig.28. response time for different request type

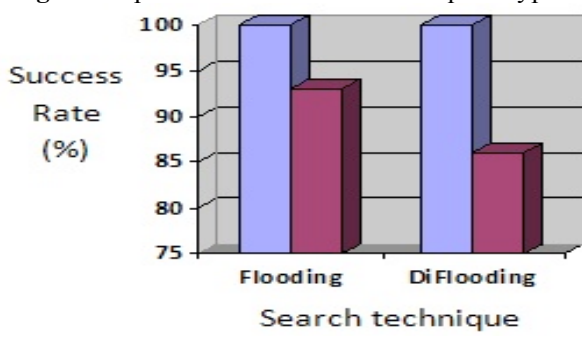

\begin{tabular}{|l|}
\hline Periodic \\
Concurrent
\end{tabular}

Fig.29. Success rate for each request type

We also study the behavior of the two techniques in different resource replication scenarios. The goal of this evaluation is to verify the impact on Flooding and DiFlooding search methods of a network having redundant resources. We measure the generated overhead, consumed battery power, resource search time and query success rate of the resource discovery protocols. We validate both these techniques with zero, i.e., no replication, five and nine redundant resource copies for a sample of 80 nodes and measure the performance metrics, as shown in Fig.30, Fig.31, Fig.32 and Fig.33, respectively. As seen in the graph, our proposed scheme reduces the network overhead, consumes minimum power, and requires less time to resolve the query as it generates less number of redundant messages in comparison to the flooding scheme, even if the resources are replicated. While it is observed that our proposed method is also equally good in terms of success rate.

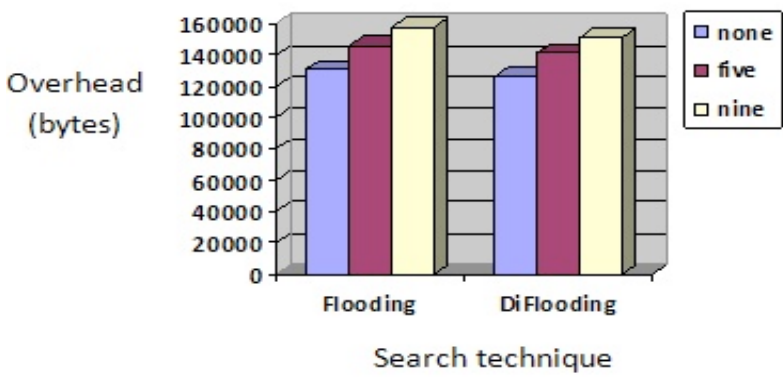

Fig.30. P2P application overhead 


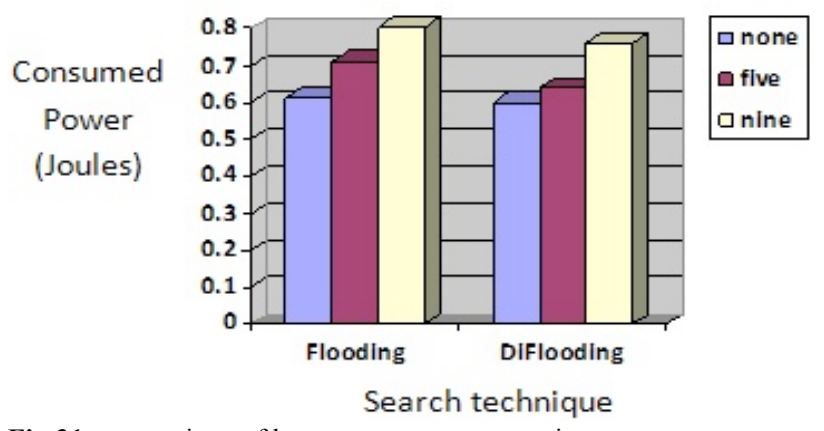

Fig.31. comparison of battery power consumption

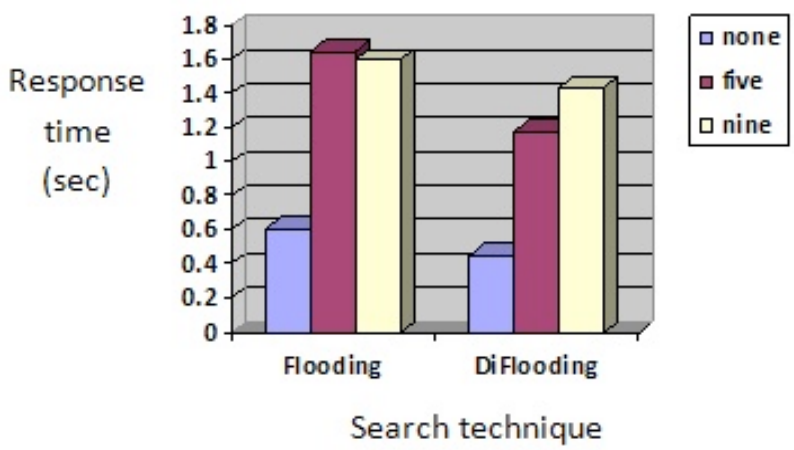

Fig.32. comparison of search delay

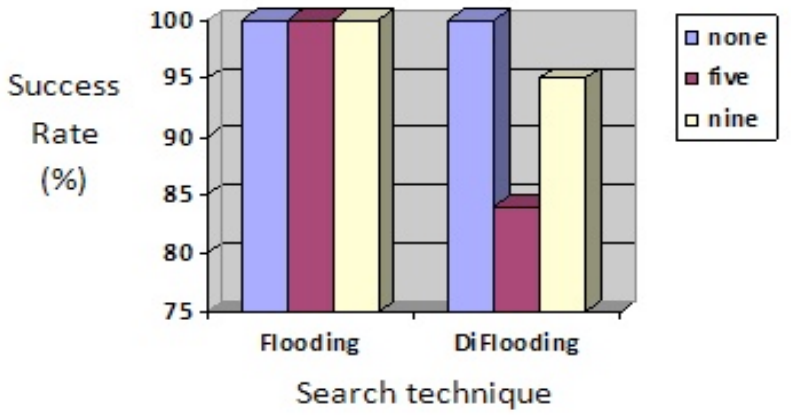

Fig.33. comparison of success rate
From the results, we conclude that even with high network load our proposed density flooding method is the best. Further our protocol lowers the utilization of network and mobile resources as compared to the flooding scheme. While the generated overhead is also less even if the resources are replicated at different nodes.

\section{Conclusion}

Broadcasting by flooding is a simple yet powerful technique for resource discovery. Many famous protocols practically used in the Internet and mobile network are using flooding in their design irrespective of the overhead since it is reliable and fast. In MANETs the distribution of nodes is random, considering node density will help improve the resource discovery process. To improve the performance and reduce overhead, we propose an optimal density based flooding search scheme and further extend our base model to propose an adaptive method that also uses the neighbor density knowledge along with local node density information to utilize the scarce mobile and network resources more efficiently in such limited environment. In this paper, we evaluate the flooding, probabilistic flooding and our proposed techniques under a highly dynamic mobile peer-to-peer network. We compare the performance of various protocols based on the metrics like network overhead, MAC load, NRL, energy consumed, success rate, query response time, bandwidth consumption, end-to-end delay, packet delivery ratio and dropped packets. From the experimental results, we conclude that our algorithms are efficient than the widely used protocols. We also verify the performance of our proposed algorithm under varying network dynamics and network loads, different network conditions, and observe that our density based scheme outperform flooding to alleviate the broadcast storm problem. The results show that our proposed methods improve all the important performance metrics.

\section{References}

1. R.Schollmeier (2003). Protocol for peer-to-peer networking in mobile environments, in Proceedings of the 12th international conference on computer communication and networks, pp. 121-127.

2. S. Androutsellis-theotokis and D. Spinellis (2004). A Survey of Peer-to-Peer Content Distribution Technologies, vol. 36, no. 4, pp. 335-371.

3. H. Choi, H. Park, and M. Woo (2006). An enhanced Gnutella for ad-hoc networks, in Systems and Networks.

4. A. Arunachalam and O. Sornil (2015). An Analysis of the Overhead and Energy Consumption in Flooding, Random Walk and Gossip Based Resource Discovery Protocols in MP2P Networks, in Fifth International Conference on Advanced Computing \& Communication Technologies, pp. 292-297.

5. A. Arunachalam and O. Sornil (2015). Issues of Implementing Random Walk and Gossip Based Resource Discovery Protocols in P2P MANETs \& Suggestions for Improvement, Procedia Comput. Sci., vol. 57, pp. 509-518.

6. Y. Tseng, S. Ni, Y. Chen, and J. Sheu (2002). The broadcast storm problem in a mobile ad hoc network, Wireless. Networks, pp. $153-167$.

7. Y. Sasson, D. Cavin, and A. Schiper (2003). "Probabilistic broadcast for flooding in wireless mobile ad hoc networks," WCNC.
8. B. Williams and T. Camp (2002). Comparison of broadcasting techniques for mobile ad hoc networks, Proc. 3rd ACM Int...., pp. 194-205.

9. V. Kalogeraki (2002). A local search mechanism for peer-topeer networks.

10. V. Dimakopoulos and E. Pitoura (2006). On the performance of flooding-based resource discovery, Parallel Distrib. Syst. IEEE Trans. vol. 17 , no. 11 , pp. $1242-1252$.

11. Z. Zhuang, Y. Liu, L. Xiao, and L. Ni (2003). Hybrid periodical flooding in unstructured peer-to-peer networks, Parallel Process.

12. Q. Lv, P. Cao, E. Cohen, K. Li, and S. Shenker (2002). Search and replication in unstructured peer-to-peer networks, in Proceedings of the 16th, pp. 84-95.

13. I. Pu and Y. Shen (2009). Enhanced blocking expanding ring search in mobile ad hoc networks, in NTMS'09, pp. 445-449.

14. R. Gaeta and M. Sereno (2011). Generalized Probabilistic Flooding in Unstructured Peer-to-Peer Networks, Parallel Distrib. Syst. IEEE Trans., vol. 22, no. 12, pp. 2055-2062.

15. H. Barjini, M. Othman, H. Ibrahim, and N. I. Udzir (2013). HybridFlood: minimizing the effects of redundant messages and maximizing search efficiency of unstructured peer-to-peer networks, Cluster Comput., vol. 17, no. 2, pp. 551-568.

16. S. Jiang, L. Guo, and X. Zhang (2003). Lightflood: an efficient flooding scheme for file search in unstructured peer-to-peer 
systems, in Parallel Processing, pp. 1-9.

17. S. Jiang, L. Guo, X. Zhang, and H. Wang (2008). Lightflood: Minimizing redundant messages and maximizing scope of peerto-peer search, Parallel Distrib. , vol. 19, no. 5, pp. 601-614.

18. T. Lin, S. Member, P. Lin, S. Member, H. Wang, and C. Chen (2009). Dynamic Search Algorithm in Unstructured Peer-to-Peer Networks, Parallel Distrib. Syst. IEEE Trans., vol. 20, no. 5, pp. 654-666.

19. M. Shojafar, J. H. Abawajy, Z. Delkhah, A. Ahmadi, Z. Pooranian, and A. Abraham (2013). An efficient and distributed file search in unstructured peer-to-peer networks, Peer-to-Peer Netw. Appl., vol. 8, no. 1, pp. 120-136.

20. T. Kato, N. Ishikawa, H. Sumino, and J. Hjelm (2003). A platform and applications for mobile peer-to-peer communications.

21. S. Doria and M. Spohn (2009). A multicast approach for peerto-peer content distribution in mobile ad hoc networks, in Wireless Communications and Networking Conference, WCNC IEEE, pp. $1-6$

22. A. Klemm (2003). A special-purpose peer-to-peer file sharing system for mobile ad hoc networks, Conf. VTC ..., pp. 27582763.

23. I. Gruber (2004). Performance evaluation of the mobile peer-to- peer service, in Proceedings of IEEE symposium on cluster computing and grid, pp. 363-371.

24. L. Zhang and J. Liu (2012). Efficient Search Scheme in Mobile Peer-to-Peer Network, in International Conference on Industrial Control and Electronics Engineering, pp. 1235-1238.

25. J. Liu (2012). The Research of Resource Searching Algorithm in Mobile P2P Network, in Fourth International Conference on Computational and Information Sciences, no. 1, pp. 811-814.

26. M. Gerla, C. Lindemann, and A. Rowstron (2005). P2P MANET's-New Research Issues., in Peer-to-Peer Mobile Ad Hoc Networks, of Dagstuhl Seminar Proceedings, Internationales Begegnungs- und Forschungszentrum für Informatik (IBFI), Schloss Dagstuhl, Germany, volume 05152, pp. $1-25$.

27. D. N. da Hora, D. F. Macedo, L. B. Oliveira, I. G. Siqueira, A. a. F. Loureiro, J. M. Nogueira, and G. Pujolle (2009). Enhancing peer-to-peer content discovery techniques over mobile ad hoc networks, Comput. Commun., vol. 32, no. 13-14, pp. 14451459.

28. M. Fathy, K. Rahemifar, H. Babaei, and R. Berangy (2012). Impact of mobility on performance of P2P content discovery protocols over MANET, in Procedia Computer Science, vol. 10, pp. 642-649. 